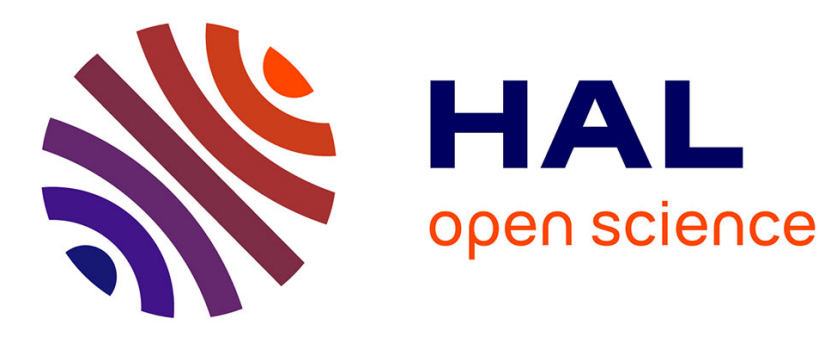

\title{
Efficacité de la maintenance conditionnelle sur des structures à dégradation aléatoire
}

\author{
Franck Schoefs, Denys Breysse, Emma Sheils, Alan O'Connor
}

\section{To cite this version:}

Franck Schoefs, Denys Breysse, Emma Sheils, Alan O'Connor. Efficacité de la maintenance conditionnelle sur des structures à dégradation aléatoire. European Journal of Environmental and Civil Engineering, 2008, 10.1080/19648189.2008.9693075 . hal-01007050

\section{HAL Id: hal-01007050 https://hal.science/hal-01007050}

Submitted on 10 Feb 2018

HAL is a multi-disciplinary open access archive for the deposit and dissemination of scientific research documents, whether they are published or not. The documents may come from teaching and research institutions in France or abroad, or from public or private research centers.
L'archive ouverte pluridisciplinaire HAL, est destinée au dépôt et à la diffusion de documents scientifiques de niveau recherche, publiés ou non, émanant des établissements d'enseignement et de recherche français ou étrangers, des laboratoires publics ou privés. 


\title{
Efficacité de la maintenance conditionnelle sur des structures à dégradation aléatoire
}

\author{
Franck Schoefs* - Denis Breysse** \\ Emma Sheils*** _ Alan O'Connor***
}

* Université de Nantes

Institut de Recherche en Génie Civil et Mécanique (GeM)

UMR 6183 CNRS, Faculté des Sciences et des Techniques

2, rue de la Houssinière

F-44000 Nantes, France

franck.schoefs@univ-nantes.fr

** Université Bordeaux1, Ghymac, France

*** Trinity College, Dublin, Ireland

RÉSUMÉ. Les stratégies de maintenance basées sur les résultats d'inspection présentent une alternative intéressante pour la gestion de structures soumises à des dégradations. L'efficacité économique et/ou technique dépend d'un grand nombre de facteurs comme la disponibilité, le coût et l'efficacité de techniques d'inspection, les mécanismes gouvernant la perte de performance, le niveau requis de service et les coûts directs et indirects. Cet article étudie, sur la base de simulations de Monte-Carlo, l'optimisation de la période entre inspections.

ABSTRACT. Inspection based maintenance strategies can provide an efficient alternative for civil engineering components subjected to ageing and degradation. The technical and/or economic efficiency of such strategies depends on many factors such as the availability, cost and efficiency of inspection techniques, the mechanisms involved in the loss of performance, the relation between what can be measured through inspections and the level of performance of the structure, the level of required serviceability of the structure, and the direct and indirect economic losses due to a reduction in the performance of a structure. On this basis, this paper studies, using Monte Carlo simulations, the benefits and limitations of an inspection based maintenance strategy.

MOTS-CLÉS: inspection, maintenance, réparation, PoD, PFA, fiabilité, analyse de risque.

KEYWORDS: inspection, maintenance, repair, PoD, PFA, reliability, risk analysis. 


\section{Introduction}

La maintenance des infrastructures ouvre la voie à de nombreuses recherches depuis les années 1980. L'objectif des travaux récents est de produire des modèles prédictifs et des simulations permettant d'optimiser les budgets d'Inspection Maintenance Réparation (IMR) (Estes et al., 1999 ; Kong et al., 2005 ; Lauridsen et al., 2006 ; Radojicic et al., 2001 ; Stewart et al., 2006). Par rapport à ces approches, cet article met en place le cadre d'une méthode générique et paramétrique qui intègre la plupart des sources d'aléas et d'incertitudes que l'on rencontre dans la pratique : le caractère aléatoire de l'évolution temporelle de la dégradation (qui dépend du matériau et des conditions d'exposition), le caractère incertain des résultats d'inspection (qui résulte de la qualité des techniques et de leur sensibilité aux biais environnementaux), le caractère aléatoire de la défaillance. On présente d'abord une modélisation probabiliste des résultats d'inspection, qui guide la politique IMR. Les modèles d'évolution, d'inspection et de coût sont proposés dans les deux sections suivantes. L'article se conclut sur une illustration dont l'objectif est l'optimisation de la périodicité des inspections.

\section{Concepts de base pour la modélisation probabiliste des résultats d'inspection}

Dans les années 1990, de nombreux travaux ont développé des concepts intégrant les imperfections dans la capacité de détection des techniques de contrôles non destructifs (CND). Parmi eux, le concept de probabilité de détection $P o D-$ probabilité de détecter un défaut existant - a connu une utilisation importante dans la maintenance des structures, donnant naissance aux stratégies de planification des inspections basées sur des analyses de risque (RBI : Risk Based Inspection) (Faber, 2002). Les secteurs les plus avancés dans cette utilisation sont les secteurs maritimes pour lesquels les coûts et les difficultés d'inspection sont des facteurs importants. Les modèles reposent soit sur des statistiques issues de campagnes d'intercalibration (Barnouin et al., 1993)), soit sur des hypothèses sur la modélisation par la théorie du signal de données d'inspection (Schoefs et al., 2007). Plus récemment, on a montré (Rouhan et al., 2003) comment la probabilité de fausse alarme $P F A$ - probabilité de détecter un défaut non existant - influe sur la politique de maintenance. Dans ce cas, des combinaisons de techniques permettent de déterminer des optimums intéressants du point de vue analyse de risque (Schoefs et al., 2004).

Dans cet article, le défaut $d(t)$ en un point est supposé exister mais sa valeur exacte est inconnue. L'inspection en fournit une estimation bruitée $\hat{d}(t)$ (équation[1]). Afin de simplifier les illustrations et de limiter le nombre de paramètres, le bruit $\eta$ est considéré comme centré ; il est aisé d'étendre le modèle au cas d'un biais de mesure. On suppose ici que le bruit est indépendant de la taille du défaut mesuré, ce qui est le cas lorsque la qualité d'inspection est fortement affectée par les conditions environnantes (visibilité, difficulté d'accès et de mise en œuvre, 
etc.). Cette hypothèse a été validée à partir d'une campagne de mesures dans le secteur portuaire (Schoefs et al., 2007).

$$
\hat{d}=d+\eta
$$

Par ailleurs, il est important de distinguer les grandeurs relatives à la calibration de techniques de contrôles non destructifs $(P o D$ et $P F A)$ et les termes relatifs à l'aide à la décision. A titre d'exemple, dans le premier cas on cherchera à déterminer la probabilité de détection d'un défaut existant $(P o D)$ alors que le décideur voudra évaluer la probabilité d'existence d'un défaut sachant qu'une détection a été obtenue. Des relations existent entre ces grandeurs à condition d'introduire un terme supplémentaire : la probabilité d'existence du défaut (Rouhan et al., 2003). Par souci de simplification, ce paramètre n'est pas introduit ici. On utilise donc les grandeurs relatives à la calibration comme support direct d'aide à la décision. Dans le cas contraire, il suffit de remplacer les termes $P o D$ et $P F A$ dans les équations [11] à [13] par les grandeurs relatives à l'aide à la décision. Ces aspects sont détaillés dans une autre publication (Sheils et al., 2007).

\section{Modélisation probabiliste de la détérioration et des inspections}

\subsection{Modèle de détérioration et de défaillance}

Le modèle d'évolution temporelle de la taille du défaut est un modèle stochastique. La croissance de la taille au cours d'un pas de temps $\Delta t$ se fait par des sauts aléatoires plus ou moins intenses. On définit alors une mesure de probabilité associée à la croissance de la taille du défaut (la taille du défaut demeure constante avec une probabilité complémentaire) (équation [2]). Une telle formulation permet de décrire, via la valeur du coefficient $g$, des lois d'évolutions plutôt régulières ou plutôt brutales.

$$
\frac{d(t+\Delta t)}{d_{0}}= \begin{cases}\frac{d(t)}{d_{0}} & \text { de probabilité }\left(1-\frac{1}{g}\right) \\ \frac{d(t)}{d_{0}}(1+g \alpha) & \text { de probabilité }\left(\frac{1}{g}\right)\end{cases}
$$

où $d(t)$ est la taille du défaut à l'instant $t, d_{0}$ la taille initiale de défaut, $g$ un paramètre décrivant la mesure de probabilité, $\Delta t$ le pas de temps et $\alpha$ une variable aléatoire distribuée normalement $\mathrm{N}[\mu ; \sigma]$ décrivant la cinétique de dégradation et de support borné à gauche de sorte que $\alpha>0$. En modifiant la valeur de $g$, l'évolution du défaut est graduelle ou plus abrupte (figure 1 , avec une distribution de $\alpha: \mathrm{N}[0,01 ; 0,002]$ tronquée à gauche). 

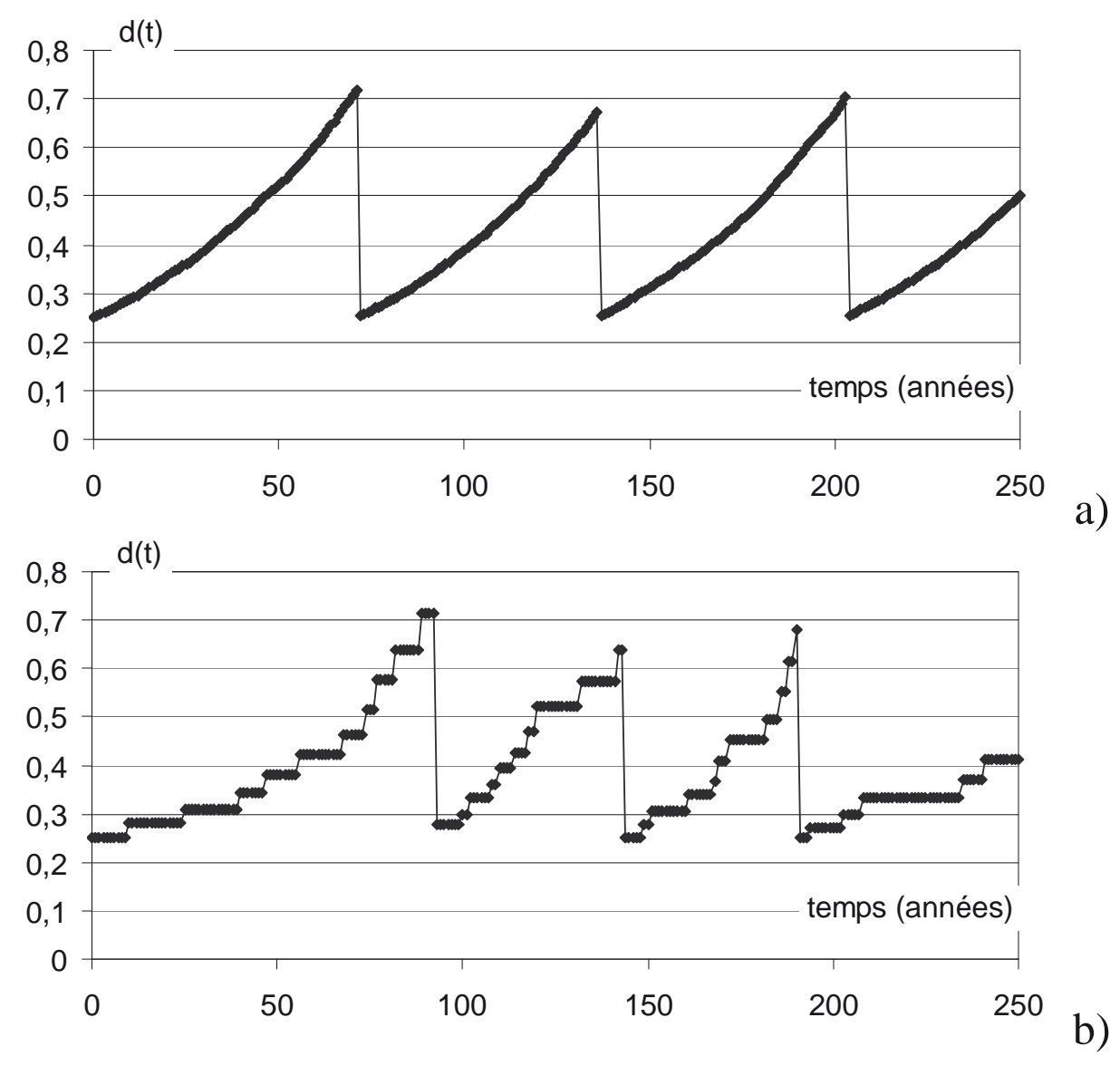

a)

Figure 1. Cinétique de détérioration avec trois cycles avec réparations $\left(d_{0}=0,25\right)$ : a) $g=1$; b) $g=7$

Pour une taille de défaut donnée, la défaillance est elle-même un événement aléatoire (dont l'occurrence dépend par exemple de la taille des actions exercées dans l'intervalle de temps considéré). La probabilité annuelle de défaillance $p_{F}$ est calculée à partir d'une fonction de répartition de Weibull (équation [3]) d'exposant $m$, décrivant l'expression de $p_{F}$ en fonction de $d(t)$.

$$
p_{F}(d(t))=1-\left[\exp -\left(\frac{d(t)-d_{1}}{d_{0}}\right)^{m}\right]
$$

La taille de défaut limite $d_{1}$ [4] correspond à une borne inférieure en deçà de laquelle on considère que la probabilité de défaillance est nulle (figure 2).

$$
p_{F}= \begin{cases}0 & d(t) \leq d_{1} \\ p_{F}(d(t)) & d(t)>d_{1}\end{cases}
$$




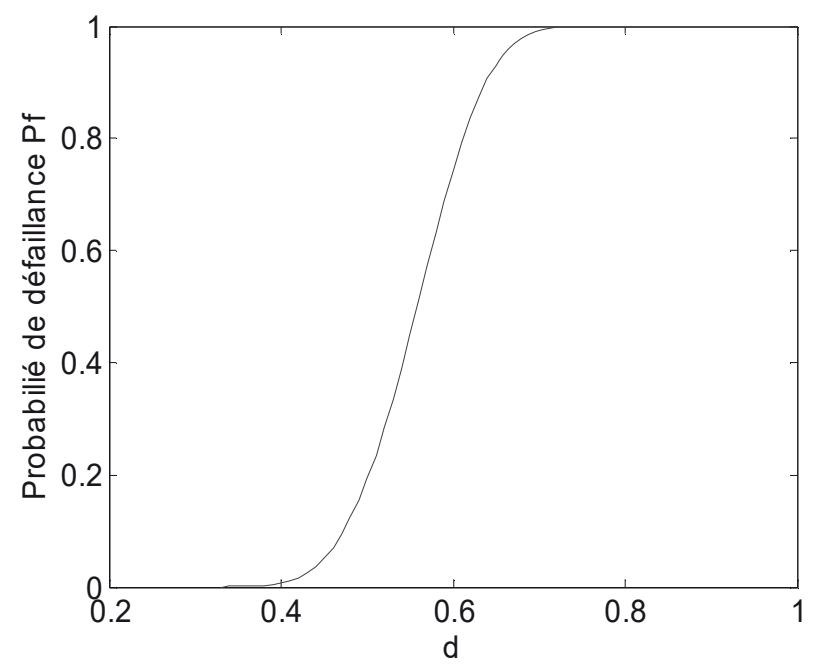

Figure 2. Evolution de la probabilité de défaillance avec la taille de défaut $\left(d_{0}=0,25 ; d_{1}=0,33 ; m=4\right)$

\subsection{Modèle d'inspection}

Le bruit $\eta$ est supposé suivre une loi normale $\mathrm{N}\left[0, \sigma_{\eta}\right]$. A partir de l'équation [1], on définit :

$$
P o D=P\left(\hat{d} \geq d_{\text {min }}\right)
$$

où $d_{\min }$ désigne le seuil de détection au-dessous duquel aucune détection n'est possible. Le modèle de $P o D$ dépend de la taille du défaut et du type d'inspection. Pour cet article on choisit une fonction de Weibull (équation [6]) d'exposant $\beta$ pour représenter la dépendance de $P o D$ avec la taille du défaut. On peut envisager des modèles d'évolution de $P F A$ en fonction de la classe de défaut. L'évaluation de cette quantité est alors très délicate. Dans l'application, on supposera que le bruit et le seuil de détection sont constants pour chaque inspection et que PFA est une constante.

$$
P o D(d(t))=1-\left[\exp -\left(\frac{d(t)-d_{\min }}{d_{0}}\right)^{\beta}\right]
$$

\subsection{Modèle de réparation}

La figure 1 illustre le cas de réparations périodiques. Une réparation est réalisée si l'on a détecté un défaut dont la taille dépasse un niveau critique $d_{c}$ calculé d'après équation [7] en fonction de $p_{a}$, la probabilité annuelle admissible de défaillance. 


$$
d_{c}=d_{1}+d_{0}\left[-\left(\ln \left(1-p_{a}\right)\right)\right]^{1 / m}
$$

En pratique, la valeur de la probabilité $p_{a}$ qui déclenche la réparation dépend du type d'état limite considéré et de la sévérité de cet état limite. On pourra par exemple distinguer des critères de type ELS, dont les conséquences sont modérées (en termes économiques, de sécurité, de perte de fonctionnalité de l'ouvrage, etc.) et des critères de type ELU, dont les conséquences seront plus sévères. A ces différents états limites correspondent des probabilités différentes, dans la mesure où l'on est plus exigeants pour la détection d'un défaut dont les conséquences risquent d'être très importantes que dans le cas d'un défaut qui n'a que des conséquences mineures. On fait aussi l'hypothèse qu'après défaillance, le défaut est systématiquement réparé. Dans le cas d'un ELU, il s'agira d'un remplacement de poutre par exemple.

\subsection{Illustration du cycle de vie d'un composant}

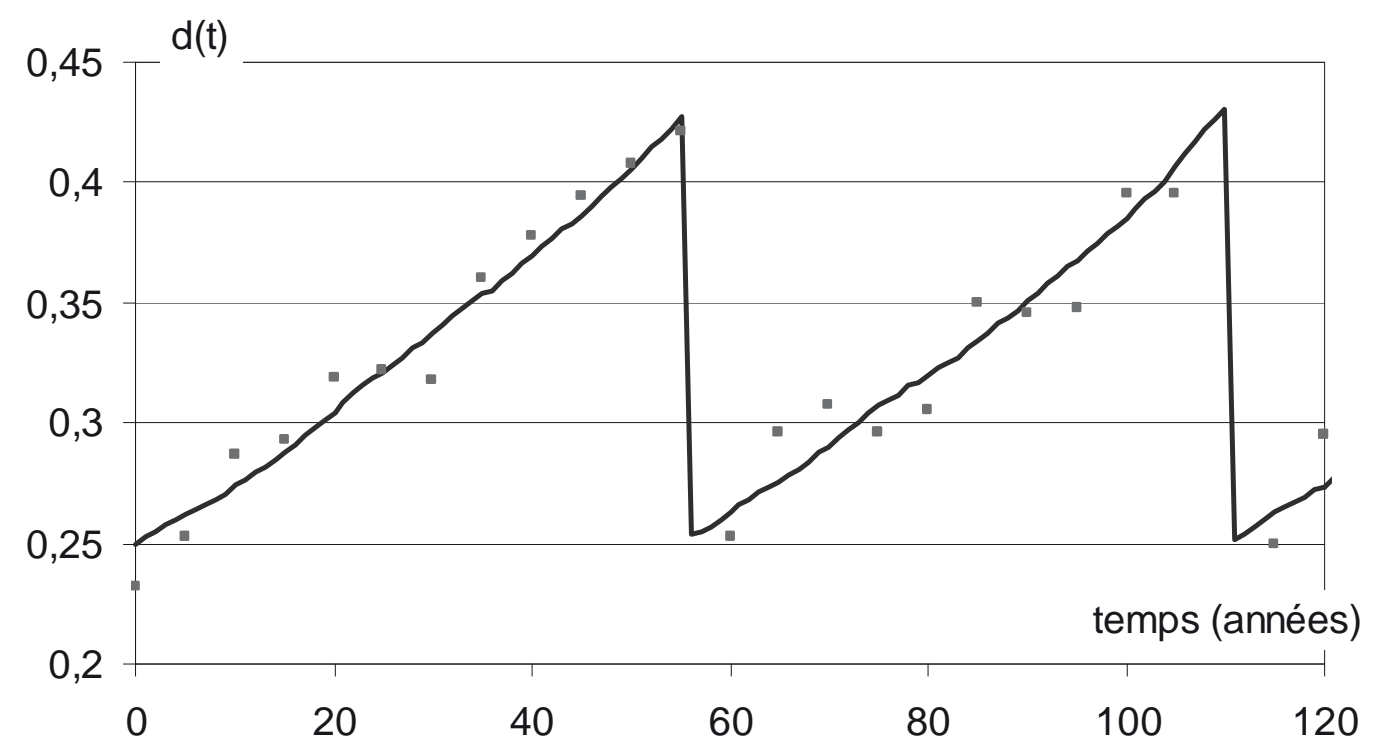

Figure 3. Cycle de vie du composant $\left(d_{0}=0,25 ; d_{c}=0,41 ; g=1\right)$

La figure 3 illustre les évolutions temporelles des défauts au cours du temps, combinant les effets de la dégradation temporelle, de l'inspection et de la réparation. On y distingue l'évolution, ici régulière, de la taille du défaut, depuis une taille initiale $d_{0}=0,25$. Les points rouges correspondent aux valeurs mesurées lors de l'inspection à laquelle on procède ici tous les 5 ans. La qualité imparfaite de la technique a pour résultat que la valeur estimée n'est pas exactement égale à la valeur vraie de la taille du défaut. Dès que la valeur estimée dépasse la taille critique (ici de l'ordre de $d_{c}=0,41$ pour une probabilité annuelle de défaillance de $1 \%$ ), on répare le composant et la taille revient à sa valeur initiale. Un autre cycle, indépendant du précédent, se développe alors. Dans le cas où la technique est de grande qualité (bruit faible), la valeur estimée se rapproche de la valeur vraie, et la décision de 
réparation est prise à bon escient. Dans le cas contraire, le résultat bruité de l'inspection conduit souvent à une réparation prématurée par rapport aux objectifs de fiabilité que l'on s'est donné a priori.

La figure 3 illustre les deux issues possibles pour les cycles. Lors du premier cycle, la valeur du $d_{c}$ est dépassée lors de l'année 51, mais la défaillance ne se produit pas et l'inspection faite l'année 55 conduit à réparer le composant. Lors du second cycle, aucune inspection n'a permis de détecter à temps le défaut qui a conduit à la défaillance à l'année 109. Pour optimiser la stratégie, il convient donc de trouver quelle est la qualité de la technique à employer, quelle est la fréquence d'inspection, mais aussi, en fonction des enjeux (en particulier des coûts induits par la défaillance), quelle est la probabilité annuelle de défaillance tolérée.

\section{Stratégies d'inspection maintenance réparation et modèles économiques}

Nous souhaitons comparer plusieurs stratégies d'inspection en conservant le mode de décision (politique de réparation) : en cas de détection de défaut, il peut y avoir réparation dans la limite du budget disponible, sinon aucune réparation n'est programmée. Des comparaisons pertinentes requièrent que l'on puisse calculer les espérances des coûts. Dans un objectif d'optimisation de la stratégie d'inspection, il s'agira de rechercher les coûts minimaux. Des modèles de coûts sont donc proposés. Selon le périmètre des actions, le coût de défaillance recouvre des conséquences indirectes : coûts sociétaux, environnementaux.

\subsection{Modèles de coût}

Le coût d'une inspection unique est calculé selon l'équation [8].

$$
C_{I}=C_{0} k_{I}\left(\frac{Q}{Q_{r e f}}\right)
$$

avec $Q$ coefficient de qualité de la technique et $k_{I}$ coefficient de pondération du coût d'inspection. La qualité de la technique conditionne directement le niveau de bruit $\sigma_{\eta}$.

Le coût de réparation en équation [9] dépend de la taille du défaut à réparer : le coût est proportionnel à un paramètre adimensionnel relié à la taille du défaut $d(t) / d_{r e f}$

$$
C_{R}=C_{0} k_{R}\left(\frac{d(t)}{d_{r e f}}\right)
$$


avec $C_{0}$ coût initial de construction, $d_{r e f}$ taille de défaut de référence et $k_{R}$ coefficient de pondération du coût de réparation. Après réparation, le défaut revient à sa taille initiale $d_{0}$.

Le coût d'une défaillance est calculé selon l'équation [10] avec $k_{F}$, coefficient de pondération du coût de défaillance.

$$
C_{F}=k_{F} C_{0}
$$

Avec une telle modélisation, trois coefficients $\left(k_{I}, k_{R}, k_{F}\right)$ permettent de décrire l'influence des poids respectifs de l'inspection, de la maintenance et de la défaillance. On peut aussi introduire un coefficient scalaire qui permet l'actualisation économique.

\subsection{Calcul des espérances mathématiques des cô̂ts}

En fonction de la taille du défaut réel, du résultat d'inspection et de la politique présentée en début de section 4, trois coûts peuvent être engendrés, auxquels contribuent, pour une certaine partie, les coûts d'inspection, de réparation et de défaillance (Sheils et al., 2007).

L'espérance mathématique de ces coûts peut être explicitée (équations [11] à [13]), pour $\mathrm{N}$ inspections à intervalles réguliers $T_{i}\left(\mathrm{~T}_{0}=0\right)$. Chacune de ces expressions est établie en explicitant les coûts conditionnels :

- le coût de l'inspection est systématique, pour chaque année d'inspection. Il est pondéré par la probabilité d'occurrence de chaque cas de manière à ce que le coût après sommation des trois cas soit $C_{I}$;

- le coût de maintenance suppose que la détection a conduit à décider que le défaut devait être réparé ;

- le coût de défaillance suppose que le défaut n'a pas été détecté - donc réparé et qu'il a conduit à la défaillance. A chaque inspection le coût de défaillance est de $C_{F} T_{i} / \Delta t$ où $\Delta t$ est le pas de temps défini en section 3.1 (en général 1 an).

On peut alors considérer trois cas selon la taille du défaut :

- premier cas : défaut trop petit pour être détecté avec la technique utilisée mais assez grand pour pouvoir provoquer la défaillance : $d<d_{\min }$ et $d_{1}<d_{\min }$

$$
\begin{gathered}
\mathrm{E}\left(\mathrm{C}_{1}(\mathrm{t})\right)= \\
\sum_{j=1}^{N} C_{I} P_{j T i}\left(d<d_{\text {min }}\right)+\sum_{j=1}^{N} C_{F} \frac{T_{i}}{\Delta t} P_{f, j T i}\left(d\left(j T_{i}\right) \mid d\left(j T_{i}\right)<d_{\text {min }}\right) P_{j T i}\left(d<d_{\text {min }}\right)
\end{gathered}
$$


- deuxième cas : défaut pouvant être détecté, mais plus petit que la taille critique déclenchant la décision de réparation : $d_{\min }<d<d_{c}$

$\mathrm{E}\left(\mathrm{C}_{2}(\mathrm{t})\right)=$

$$
\begin{aligned}
& \sum_{j=1}^{N} C_{I} P_{j T i}\left(d_{\text {min }}<d\left(j T_{i}\right)<d_{c}\right)+\sum_{j=1}^{N} C_{R} \operatorname{PFA}\left(d\left(j T_{i}\right) \mid d_{\text {min }}<d\left(j T_{i}\right)<d_{c}\right) \\
& P_{j T i}\left(d_{\text {min }}<d\left(j T_{i}\right)<d_{c}\right)+ \\
& \sum_{j=1}^{N} C_{F} \frac{T_{i}}{\Delta t}\left[\left(1-P F A\left(d\left(j T_{i}\right) \mid d_{\text {min }}<d\left(j T_{i}\right)<d_{c}\right)\right) \cdot P_{j T i}\left(d_{\text {min }}<d\left(j T_{i}\right)<d_{c}\right)\right] \\
& {\left[P_{f, j T i}\left(d\left(j T_{i}\right) \mid d_{1}<d\left(j T_{i}\right)<d_{c}\right) P_{j T i}\left(d_{1}<d\left(j T_{i}\right)<d_{c}\right)\right]}
\end{aligned}
$$

L'expression [12] tient compte de ce que le défaut peut être estimé plus grand qu'il n'est en réalité (fausse alarme) et réparé de façon trop précoce ;

- troisième cas : défaut dont la taille dépasse la taille critique : $d_{\min }<d_{c}<d$ : (on suppose $d_{1}<d_{c}$ )

$\mathrm{E}\left(\mathrm{C}_{3}(\mathrm{t})\right)=$

$$
\begin{aligned}
& \sum_{j=1}^{N} C_{I} P_{j T i}\left(d_{c}<d\left(j T_{i}\right)\right)+\sum_{j=1}^{N} C_{R}\left[\operatorname{PoD}\left(d\left(j T_{i}\right) \mid d_{c}<d\left(j T_{i}\right)\right)\right] P_{j T i}\left(d_{c}<d\left(j T_{i}\right)\right) \\
& +\sum_{j=1}^{N} C_{F} \frac{T_{i}}{\Delta t}\left[\left(1-\operatorname{PoD}\left(d\left(j T_{i}\right) \mid d_{c}<d\left(j T_{i}\right)\right) . P_{j T i}\left(d_{c}<d\left(j T_{i}\right)\right)\right]\right. \\
& {\left[P_{f, j T i}\left(d\left(j T_{i}\right) \mid d_{c}<d\left(j T_{i}\right)\right) P_{j T i}\left(d_{c}<d\left(j T_{i}\right)\right)\right]}
\end{aligned}
$$

L'expression [13] tient compte de ce que le défaut peut être estimé plus petit qu'il ne l'est en réalité (non-détection) et non réparé alors qu'il devrait l'être. Ces formules ont valeur de généralité et tiennent compte d'une dépendance de $P F A$ à la taille de défaut. Comme indiqué en section 3.2, $P F A$ sera considérée comme constante par la suite.

\section{Simulations de la stratégie d'IMR}

\subsection{Principes et objectifs de la simulation}

L'ensemble des modèles ayant été défini, on peut procéder aux simulations numériques. Les valeurs numériques choisies sont données dans le tableau 1. 


\begin{tabular}{ll} 
Taux de croissance $\alpha$, moyenne $\mu$ et écart type $\sigma$ & 0,$01 ; 0,002$ \\
Taille initiale de défaut $d_{0}$; défaut de déférence $d_{r e f}$ & 0,$25 ; 1$ \\
Paramètre de cinétique de dégradation, $g$ & 1 \\
Probabilité de défaillance & 4 \\
\hline Exposant de la probabilité de défaillance, $m$ & 0,33 \\
Taille de défaut limite, $d_{1}$ & 0,01 \\
Probabilité annuelle de défaillance acceptable, $p_{a}$ & \\
\hline Paramètres relatifs à la technique CND & 0,3 \\
Seuil de détection, $d_{\text {min }}$ & $8 ; 20$ \\
Qualité de l'inspection, $Q$ et qualité de référence $Q_{r e f}$ & 0,5 \\
PFA & \\
Paramètres relatifs aux modèles de coût & 1000 \\
Coût initial de construction, $C_{0}$ & 0,$005 ; 0,05$ \\
Coefficients d'inspection, $k_{I}$ et de réparation, $k_{R}$ & 1 \\
Coefficient d'impact de la défaillance, $k_{F}$ &
\end{tabular}

Tableau 1. Paramètres retenus pour les modèles (sans actualisation économique)

Ces paramètres conduisent, sans maintenance, à une durée de vie moyenne avant défaillance d'une soixantaine d'années. On réalise des simulations de Monte-Carlo en considérant des chroniques de 10000 ans, ce qui revient à suivre dans le temps l'évolution d'un composant pendant environ 150 à 200 cycles de vie, ou, de manière équivalente, à considérer simultanément 150 à 200 composants jusqu'à leur défaillance.

Nous allons étudier consécutivement l'effet de la durée entre deux inspections et l'effet de la qualité de la technique. Nous noterons avant tout commentaire que les comparaisons de coûts doivent être faites avec prudence, car les résultats dépendent fortement des valeurs choisies pour les trois coefficients $k_{I}, k_{R}$ et $k_{F}$.

\subsection{Intervalle optimal entre deux inspections}

Les figures 4 à 6 rassemblent les résultats obtenus si l'on fait varier l'intervalle entre deux inspections de 1 à 10 ans.

Les résultats de la figure 4 appellent plusieurs commentaires :

- le nombre de défaillances croît avec l'intervalle entre deux inspections, dans la mesure où la probabilité de défaillance croît si la durée croît, et deux inspections trop distantes ne permettent pas de prévenir cette défaillance (le défaut est sous le seuil critique lors de la première des deux inspections) ;

- le nombre de réparations faites après une fausse alarme est plus élevé que celui de réparations faites sur la base d'une bonne décision, et ce d'autant plus que l'intervalle entre deux inspections est faible. Cela provient du caractère imparfait de la technique de mesure, qui conduit à de nombreuses surestimations de la taille du défaut, et donc à 
des réparations prématurées (voir figure 3). Une conséquence directe est que la durée moyenne de service passe de moins de 40 ans $\left(38,1=10000 / 262,6\right.$ si $\mathrm{T}_{\mathrm{i}}=1$ an) à plus de 50 ans $\left(53,0=10000 / 188,6\right.$ si $\mathrm{T}_{\mathrm{i}}=10$ ans $)$.

Les coûts attachés à chaque stratégie sont comparés sur la figure 5. On y distingue les coûts d'inspection, ceux de réparation (en faisant la part de ceux dus à une fausse alarme et de ceux basés sur une bonne décision), et de ceux de défaillance. Avec les paramètres choisis, les coûts de réparation demeurent faibles et la valeur optimale de l'intervalle entre deux inspections résulte principalement du fait que, quand cet intervalle augmente, les coûts d'inspection décroissent, et les coûts de défaillance augmentent. L'optimum résultant est ici de 4 ans.

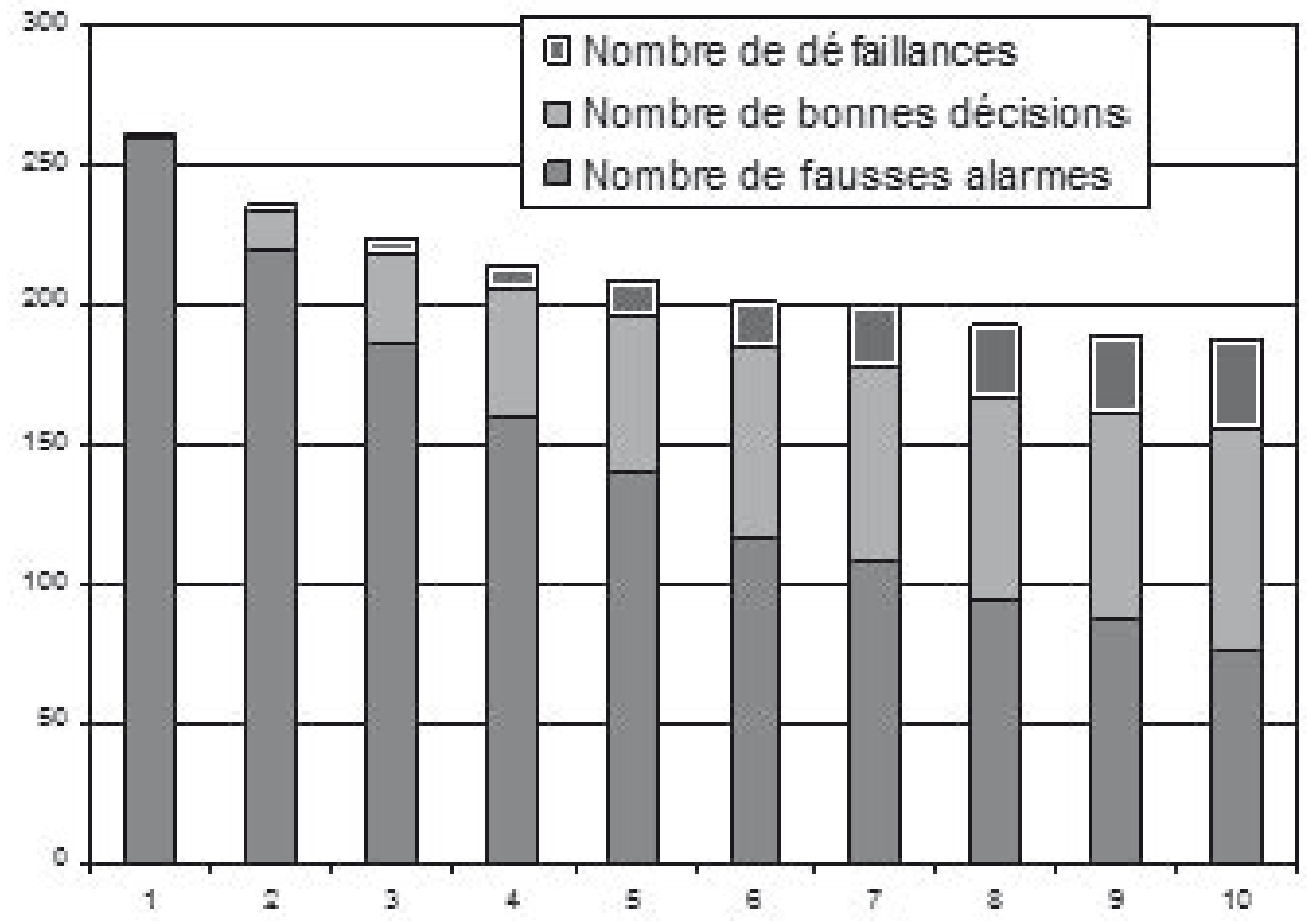

Figure 4. Effectif des causes possibles de fin de cycle en fonction de l'intervalle entre deux inspections

En comparant ces résultats avec ceux de la figure 4, on remarque que le nombre de fausses alarmes diminue avec le temps car les défauts augmentent et sont donc plus faciles à détecter (figure 4) et génère un coût des réparations diminuant avec le temps (figure 5). De manière complémentaire, le nombre de défaillances augmente avec le temps car on ne détecte pas tous les défauts même de taille importante qui induisent les défaillances et le coût de défaillance augmente alors avec le temps.

La figure 6 présente les résultats des mêmes simulations, pour une variante de simulation dans lesquels on a simplement modifié les coûts unitaires de chaque opération (tableau 2). Dans la variante, on attache un poids relatif plus important aux coûts de réparation. Le résultat est un déplacement vers de plus fortes valeurs de l'intervalle optimum entre deux inspections, avec une valeur optimale de l'ordre de 6 à 7 ans. La forme de la fonction coût montre en outre qu'elle est très sensible aux hypothèses sur le contexte économique. 


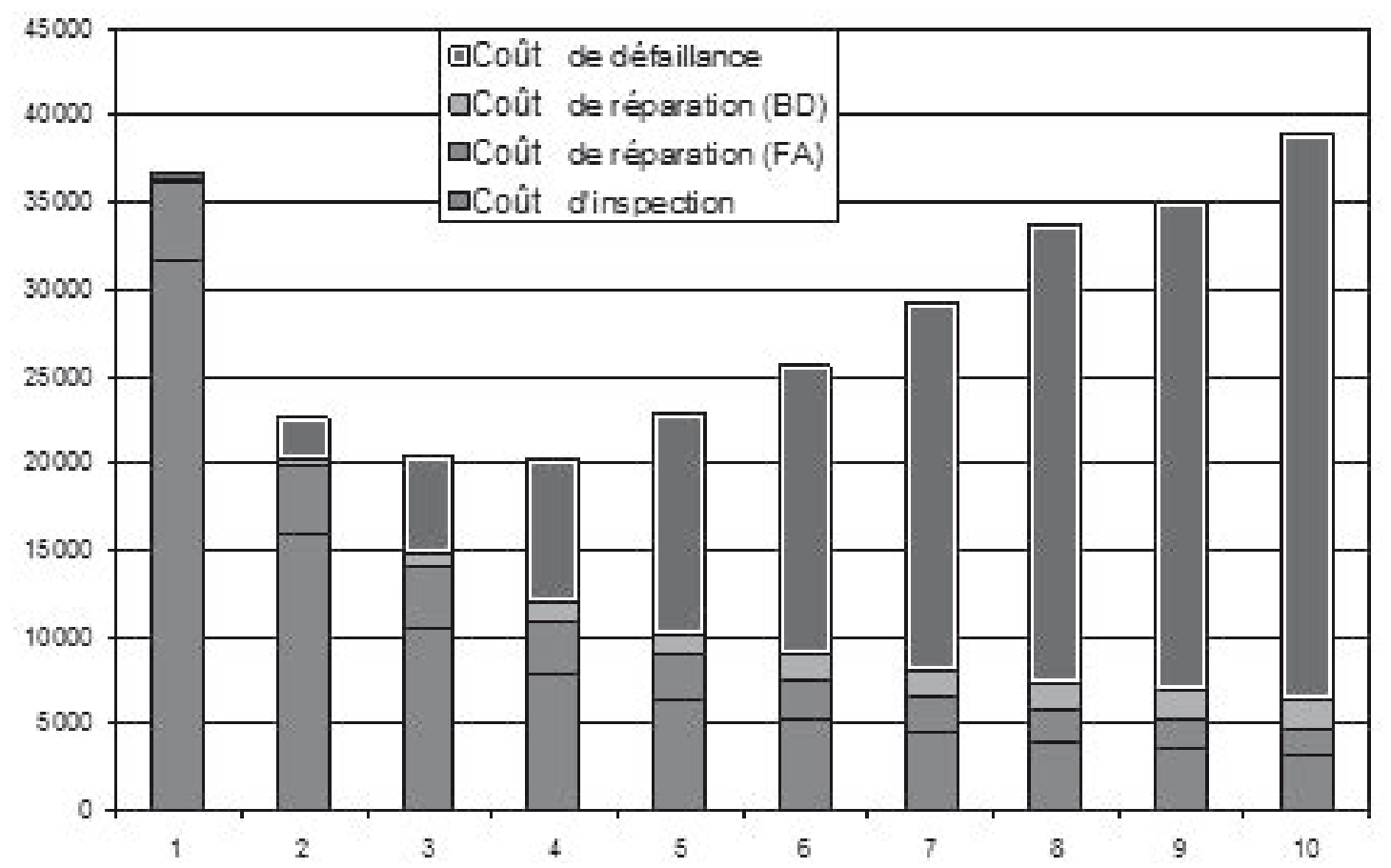

Figure 5. Variation de l'espérance des coûts en fonction de l'intervalle entre deux inspections

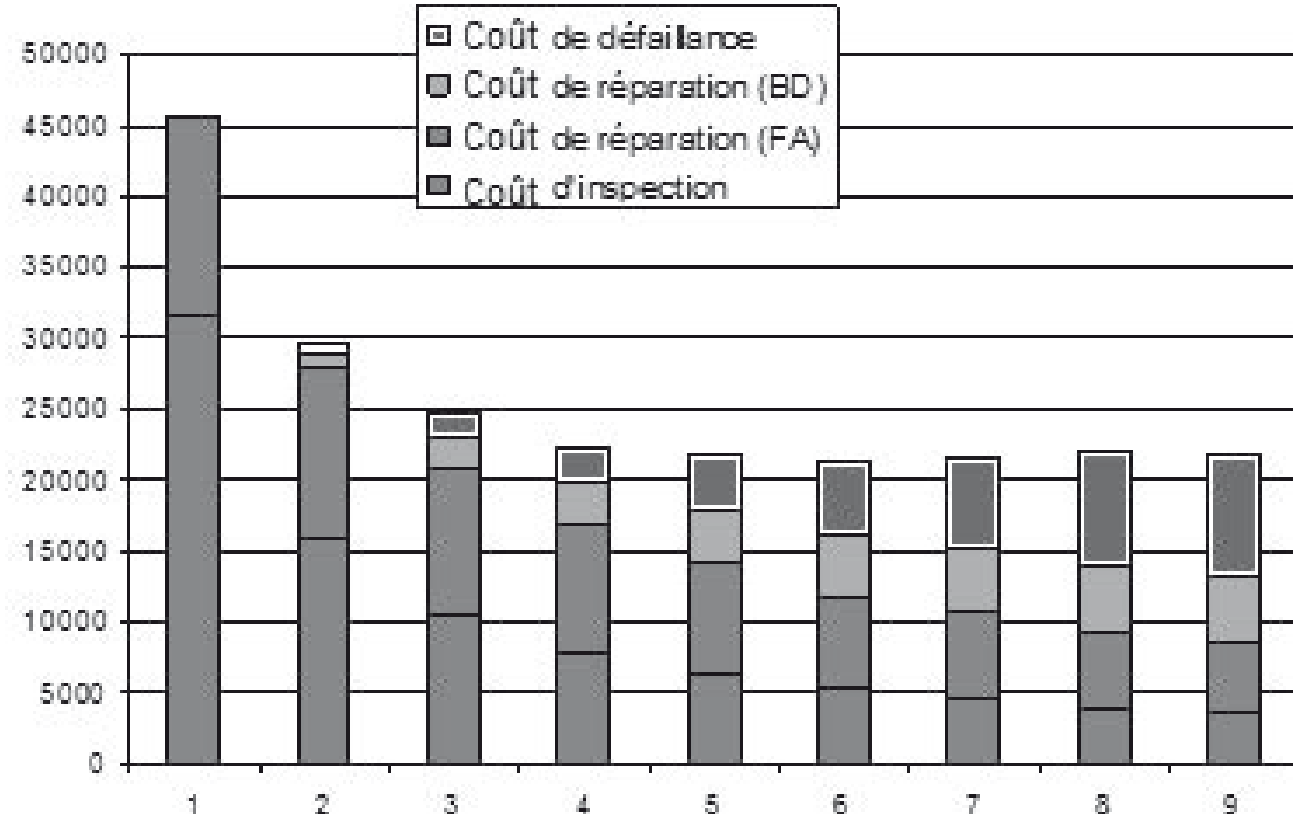

Figure 6. Variation de l'espérance des coûts en fonction de l'intervalle entre deux inspections (simulation, cas B)

\begin{tabular}{cccc}
\hline & $\begin{array}{c}\text { Simulation de référence } \\
(\text { cas A) }\end{array}$ & $\begin{array}{c}\text { Variante } \\
(\text { cas B) }\end{array}$ & $\begin{array}{c}\text { Variante } \\
(\text { cas C) }\end{array}$ \\
\hline Inspection $k_{I}$ & 0,005 & 0,005 & 0,001 \\
Réparation $k_{R}$ & 0,05 & 0,15 & 0,15 \\
Défaillance $k_{F}$ & 1 & 0,3 & 0,3 \\
\hline
\end{tabular}

Tableau 2. Coûts unitaires pour les deux cas de simulation 


\subsection{Effet de la qualité d'inspection}

La figure 7 illustre l'influence de la qualité de la technique, que l'on fait varier de $\mathrm{Q}=5$ à $\mathrm{Q}=512$. Les données des coûts sont celles du Cas $\mathrm{C}$ (tableau 2), et on considère un intervalle constant entre deux inspections $T_{i}=7$ ans.

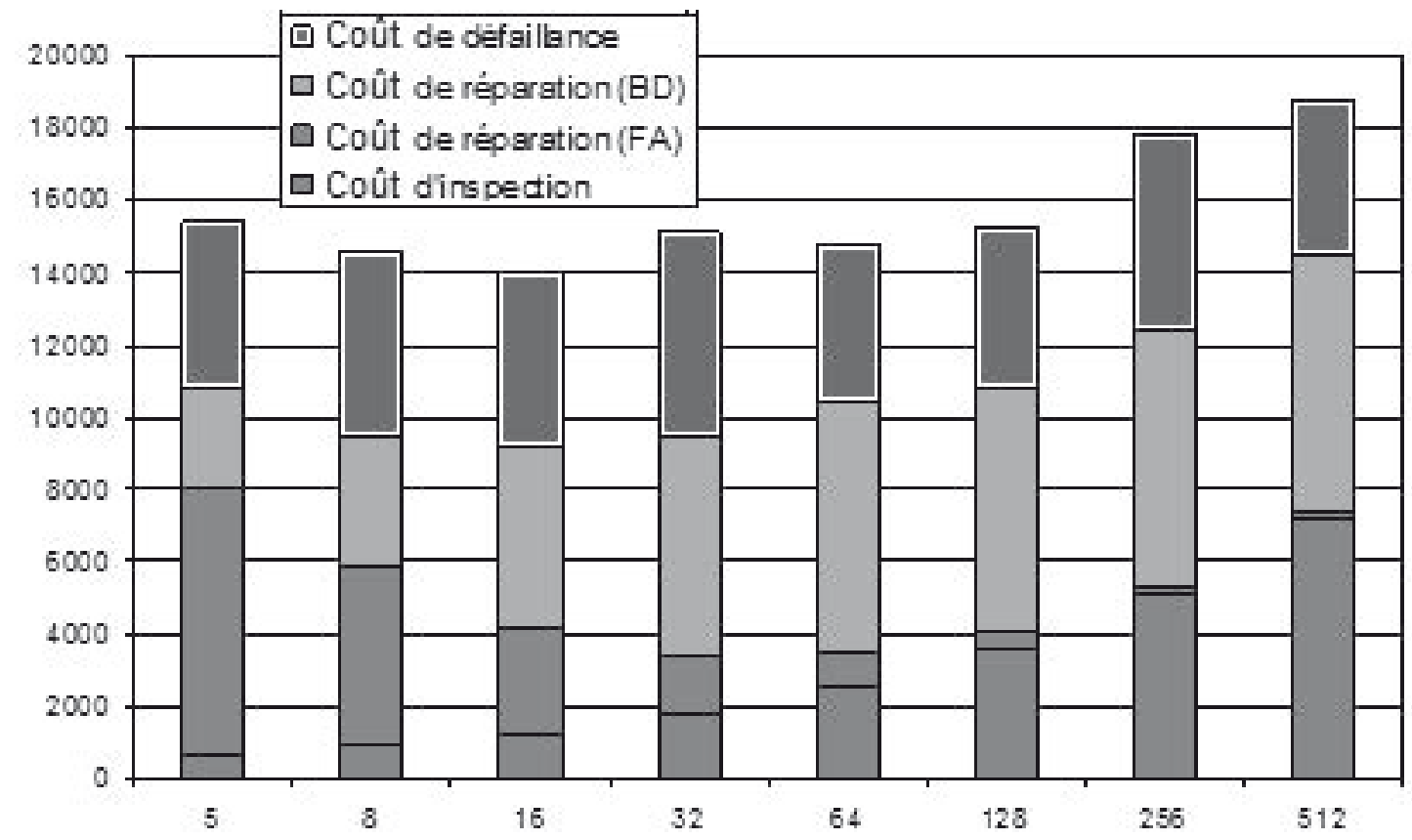

Figure 7. Influence de la qualité de la technique d'inspection sur les coûts (simulation, cas C)

La simulation montre que l'optimum correspond à une technique de qualité intermédiaire. Une technique trop sophistiquée est trop coûteuse sans diminuer les coûts de maintenance et de défaillance (pour cet intervalle et cette cinétique de développement des défauts). A l'inverse, une technique trop fruste, même peu coûteuse conduit à des réparations trop précoces et augmente les coûts correspondants.

\section{Conclusion}

Nous avons présenté ici les premiers résultats d'une approche globale de l'optimisation d'une politique d'inspection-maintenance-réparation dans un contexte où les incertitudes portent à la fois sur la croissance des défauts, sur les résultats de l'inspection et sur les possibilités de défaillance. Le formalisme général est adapté au contexte de gestion vis-à-vis d'états limites de service aussi bien que d'états limites ultimes. Il peut aussi s'appliquer à des phénomènes de dégradation très divers, même s'il a été originellement développé dans l'optique de la maintenance d'ouvrages corrodés. Les simulations permettent de comparer différentes options stratégiques, par exemple en comparant l'efficacité de différentes techniques d'inspection, ou de différentes fréquences d'inspection. 
Les résultats doivent être considérés aujourd'hui comme illustratifs, dans la mesure où ils dépendent largement des hypothèses faites à chaque étape du processus de modélisation, en particulier sur les composantes économiques. Il apparaît donc essentiel d'alimenter les modèles, aussi bien en ce qui concerne les capacités des techniques, l'efficacité des réparations, que de données sur les coûts. Le projet SENSO (2006-2008), financé par l'ANR (Agence nationale de la recherche), qui a pour objectif d'établir une base de données sur la qualité des techniques de CND (intensité des bruits, sensibilité des techniques, etc.), contribue ainsi par exemple à alimenter la modélisation des résultats d'inspection d'ouvrages en béton armé. Le projet MAREO (2008-2010), financé par le FCE (Fonds de compétitivité des entreprises) et la région Pays de la Loire, vise quant à lui à fournir des modèles d'efficacité de réparations d'ouvrages en béton armé.

Une autre perspective est de développer des modèles décomposant la stratégie d'acquisition d'information sur l'ouvrage en distinguant la phase de détection des défauts de la phase de qualification des défauts (Sheils et al., 2007 ; Breysse et al., 2008).

\section{Remerciements}

Cette étude est menée dans le cadre du projet européen MEDACHS, financé par les fonds FEDER (Interreg IIIb), et dédié à l'analyse de la maintenance et de la fiabilité de structures vieillissantes placées en environnement marin.

\section{Bibliographie}

Barnouin B., Lemoine L, Dover W.D., Rudlin J., Fabbri S., Rebourcet G., Topp D., Kare R., Sangouard D., "Underwater inspection reliability trials for offshore structures", in ASME NY (ed.), Proc. of the $12^{\text {th }}$ OMAE Conference, vol. 2, 1993, p. 883-890.

Breysse D., Elachachi S.M., Sheils E., Schoefs F., "Life cycle cost analysis of ageing structural components based on non destructive condition assessment", Australian Journal of Structural Engineering, Special Issue Engineering Decision Making issue, M. Stewart and S. Reid (guest ed.), 2008.

Estes A.C., Frangopol D.M., "Repair optimization of highway bridges using system reliability approach", Journal of Structural Engineering, Journal of IABSE, vol. 9, n 3, July 1999, p. 219-223.

Faber M.H., "RBI: An introduction", Structural Engineering International, vol. 3, 2002, p. 187-194.

Kong J.S., Frangopol D.M., "Probabilistic optimization of ageing structures considering maintenance and failure costs", Journal of Structural Engineering, vol. 131, n ${ }^{\circ} 4$, April 2005, p. 600-616. 
Lauridsen J., Bjerrum J., Sloth M., Jensen F.M., "Principles for a guideline for probabilitybased management of deteriorated bridges", IABMAS 2006, Porto, Portugal, July 16-19, 2006.

Onoufriou T., Frangopol D.M. "Reliability-based inspection optimisation of complex structures: a brief retrospective", Computers \& Structures, Pergamon, vol. 80, $\mathrm{n}^{\circ} 12$, 2002, p. 1133-1144.

Radojicic A., Bailey S.F., Brühwiler E., "Probabilistic models of cost for the management of existing structures", Life-Cycle Cost Analysis and Design of Civil Infra-structure Systems, Structural Engineering International, Journal of IABS, August 2001, p. 251-270.

Rouhan, A., Schoefs F. "Probabilistic modelling of inspection results for offshore structures", Journal of Structural Safety, vol. 25, 2003, p. 379-399.

Rudlin J.R., Dover W.D., "The ICON database: Assisting underwater inspections", in: Offshore Technology, vol. 4, 1996.

Schoefs F., Clément A., "Multiple inspections modeling for decision making and management of jacket offshore platforms: effect of false alarms", International Forum on Engineering Decision Making, Switzerland, 2004.

Schoefs F., Clément A., Memet J.B., Nouy A., "Spatial dependence of receiver operating characteristic curves for Risk Based Inspection of corroded structures: application to onpile wharf', Proceeding of I.C.A.S.P'07, Tokyo, Japan, July 31-August 32007.

Sheils E., Schoefs F., Breysse D., O’Connor A., "Comparing efficiency of systematic and conditional maintenance for randomly ageing components", Proceedings of I.C.A.S.P'07, Tokyo, Japan, July 31-August 32007.

Stewart M.G., Mullard J.A., "Reliability based assessment of the influence of concrete durability on the timing of repair for RC bridges", IABMAS 2006, Porto, Portugal, July 16-19, 2006. 